${ }^{1}$ Graduate Program in Dentistry, Meridional College/IMED, Passo Fundo, Brazil.

${ }^{2} \mathrm{MSciD}$ and PhD Graduate Program in Oral Science, Faculty of Dentistry, Federal University of Santa Maria (UFSM), Santa Maria, Rio Grande do Sul State, Brazil.
Corresponding author: Rafael Sarkis-Onofre Graduate Program in Dentistry Meridional Faculty/IMED, 304, Senador Pinheiro Street Passo Fundo - Brazil

99070-220

e-mail: rafael.onofre@imed.edu.br

Received: October 26, 2020

Accepted: December 16, 2021

\section{How are meta-analyses being conducted and reported in dentistry? a meta-research study}

Letícia Tainá de Oliveira Lemes ${ }^{1}$ (D), Lara Dotto ${ }^{1}$ (D), Bernardo Antonio Agostini ${ }^{1}$ (D, Gabriel Kalil Rocha Pereira $^{2}$ (D), Rafael Sarkis-Onofre ${ }^{1, *}$ (1)
Aim: This study aimed to evaluate how meta-analyses are conducted and reported in dentistry. Methods: We conducted a search to identify dentistry-related Systematic Reviews (SRs) indexed in PubMed in 2017 (from January 01 until December 31) and published in the English language. We included only SRs reporting at least one meta-analysis. The study selection followed the 4-phase flow set forth in the Preferred Reporting Items for Systematic Reviews and Meta-Analyses Statement (PRISMA) and it was independently conducted by two researchers. Data extraction was performed by one of three reviewers, and data related to conducting and reporting of the meta-analysis were collected. Descriptive data analysis was performed summarizing frequencies for categorical items or median and interquartile range for continuous data. Results: We included 214 SRs with meta-analyses. Most of the studies reported in the title that a meta-analysis was conducted. We identified three critical flaws in the included studies: Ninety (90) meta-analyses (43.1\%) did not specify the primary outcome; most of the meta-analyses reported that a measure of statistical heterogeneity was used to justify the use of a fixed-effect or random-effects meta-analysis model $(n=114,58.5 \%)$; and a great part did not assess publication bias ( $n=106,49.5 \%)$. Conclusion: We identified deficiencies in the reporting and conduct of meta-analysis in dentistry, suggesting that there is room for improvement. Educational approaches are necessary to improve the quality of such analyses and to avoid biased and imprecise results.

Keywords: Oral health. Systematic reviews as topic. Research report. 


\section{Introduction}

Meta-analyses are an important component of systematic reviews and are a statistical method to combine results from two or more independent studies ${ }^{1}$. This method enables improving the precision of estimates and answering conflicting questions or questions not discussed in individual studies². However, meta-analyses might be misused and biased in a similar way to other research methods.

loannidis ${ }^{3}$ (2016) demonstrated that 9,135 meta-analyses were published in PubMed in 2014, corresponding to an increase in the publication rate of 2,635\% between 1991 and 2014. The author highlighted that many meta-analyses are redundant, unnecessary, or have methodological flaws. Page et al. ${ }^{4}$ investigated flaws in the application and interpretation in a sample of meta-analyses of therapeutic interventions. The findings demonstrated problems in aspects such as interpretation of the model used, subgroup analyses, and the minimum number of studies recommended to test funnel plot asymmetry.

In dentistry, Saltaji et al. ${ }^{5}$ assessed 1,118 systematic reviews (SRs) published between 1991-2012; the findings demonstrated that almost 50\% performed a meta-analysis, and the median of included studies in the largest meta-analysis was 9. Moreover, several articles assessed the quality of meta-analyses performed in different oral health specializations, demonstrating that the overall quality varies from low to medium ${ }^{6}$. However, there are no studies in dentistry evaluating reporting and conducting meta-analysis characteristics, which would enable highlighting areas for future improvement. Thus, this study aimed to evaluate how meta-analyses are conducted and reported in dentistry.

\section{Materials and Methods}

We used a dataset of SRs in dentistry indexed in PubMed in 2017. The reporting and conduct characteristics of these SRs were previously published7. We have only evaluated data from SRs with meta-analysis and assessed how meta-analyses are conducted and reported.

\section{Search and Eligibility criteria}

A full description of the search strategy and eligibility criteria is available in the study of Bassani et al. (2019)7. First, we conducted a search in PubMed to identify dentistry-related SRs published in the English language indexed in 2017 (from January 01 until December 31). We considered the article as a SR based on the Preferred Reporting Items for Systematic reviews and Meta-Analysis Protocols (PRISMA-P) definition ${ }^{8}$. In this article, we have only included SRs reporting at least one meta-analysis. In addition, we included articles regardless of questions answered and study designs included (clinical studies, in vitro, in situ, etc.). The search strategy used is presented in Table 1 and was based on MeSH terms of PubMed and a specific filter (U.S. National Library of Medicine).

\section{Screening}

We selected the studies using a reference manager software (EndNote $X 7$, Thomson Reuters, New York, USA). Details about the pilot test screening and study selection are 
“Oral Health"[Mesh] OR “Oral Health" OR “Health, Oral” OR "Dentistry"[Mesh] OR “Dentistry" OR “Dental Research"[Mesh] OR "Dental Research" AND (((systematic review [ti] OR meta-analysis [pt] OR meta-analysis [ti] OR systematic literature review [ti] OR this systematic review [tw] OR pooling project [tw] OR (systematic review [tiab] AND review [pt]) OR meta synthesis [ti] OR meta synthesis [ti] OR integrative review [tw] OR integrative research review [tw] OR rapid review [tw] OR consensus development conference [pt] OR practice guideline [pt] OR drug class reviews [ti] OR cochrane database syst rev [ta] OR acp journal club [ta] OR health technol assess [ta] OR evid rep technol assess summ

[ta] OR jbi database system rev implement rep [ta]) OR (clinical guideline [tw] AND management [tw]) OR ((evidence based[ti] OR evidence-based medicine [mh] OR best practice* [ti] OR evidence synthesis [tiab]) AND (review [pt] OR diseases category[mh] OR behavior and behavior mechanisms [mh] OR therapeutics [mh] OR evaluation studies[pt] OR validation studies[pt] OR guideline [pt] OR pmcbook)) OR ((systematic [tw] OR systematically [tw] OR critical [tiab] OR (study selection [tw]) OR (predetermined [tw] OR inclusion [tw] AND criteri* [tw]) OR exclusion criteri* [tw] OR main outcome measures [tw] OR standard of care [tw] OR standards of care [tw]) AND (survey [tiab] OR surveys [tiab] OR overview* [tw] OR review [tiab] OR reviews [tiab] OR search* [tw] OR handsearch [tw] OR analysis [ti] OR critique [tiab] OR appraisal [tw] OR (reduction [tw]AND (risk [mh] OR risk [tw]) AND (death OR recurrence))) AND (literature [tiab] OR articles [tiab] OR publications [tiab] OR publication [tiab] OR bibliography [tiab] OR bibliographies [tiab] OR published [tiab] OR pooled data [tw] OR unpublished [tw] OR citation [tw] OR citations [tw] ,OR database [tiab] OR internet [tiab] OR textbooks [tiab] OR references [tw] OR scales [tw] OR papers [tw] OR datasets [tw] OR trials [tiab] OR meta-analy* [tw] OR (clinical [tiab] AND studies [tiab]) OR treatment outcome [mh] OR treatment outcome [tw] OR pmcbook)) NOT (letter [pt] OR newspaper article [pt])))

available in the study by Bassani et al. ${ }^{7}$. The study selection followed the 4-phase flow set forth in the Preferred Reporting Items for Systematic Reviews and Meta-Analyses Statement ${ }^{9}$. Two researchers independently identified articles by reviewing titles and abstracts, and then screening the full text in a second phase.

\section{Data extraction}

A standardized form was created using Microsoft Excel based on the study by Page et al. ${ }^{10}$ (2016). A pilot data extraction was performed to ensure consistency, and the details are available in the study by Bassani et al. ${ }^{7}$. Data related to the conduct and reporting of meta-analyses were extracted by one of three reviewers. Data regarding the journal category (general or specialized journal), number of authors, SR focus (Epidemiology, Diagnosis, Prevention, Prognosis, Treatment/ Therapeutic, Other, Unclear, or Mixed), dental specialization, funding (reported or not reported), and data related to conducting and reporting of meta-analysis such as details about the primary outcome, the model used, number of studies in the largest meta-analysis, statistical heterogeneity, publication bias, additional analysis and software used to conduct the meta-analyses were extracted. The form used is available in the supplementary material. One author subsequently verified the data consistency, and the data were extracted again in the case of any doubt or inconsistency.

\section{Data analysis}

Descriptive analysis of the data was performed by summarizing frequencies for categorical items and calculating median and interquartile range for continuous data. All analyses were performed using Stata 14.2 software. Characteristics of the meta-analyses were assessed considering all included SRs and meta-analyses. 


\section{Results}

Figure 1 presents a flow diagram outlining the study selection process. The initial search in PubMed yielded 1375 records, and we included 214 SRs with meta-analysis after study screening of the title/abstract and full-text analysis.

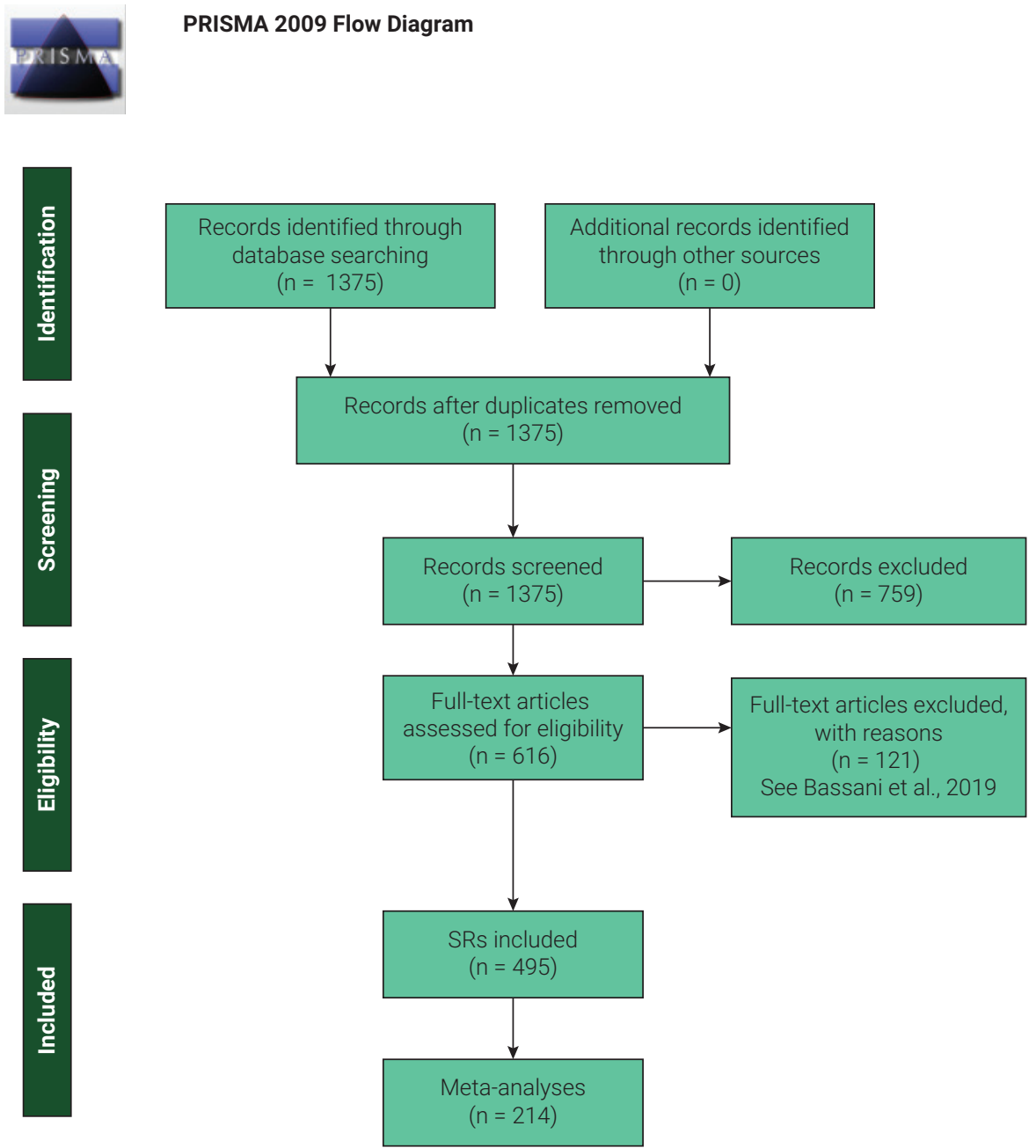

Figure 1. Flow diagram of study selection

Table 2 presents the epidemiological characteristics of the SRs with meta-analyses included in the study. Most of the meta-analyses were published in specialized journals $(n=159,74,3 \%)$. Considering the country where those meta-analyses were produced, 4 countries produced $64.3 \%$ of SR with meta-analysis published, in which Brazil had the greatest contribution with 61 (28.5\%). The main specialization was Oral and Maxillofacial Surgery ( $n=32,14.9 \%)$, followed by Implantology $(n=31,14.5 \%)$ and Periodontics $(n=29$, $13.5 \%$ ). The main focus of most of the SRs with meta-analyses was treatment/therapeutic $(n=101,47.2 \%)$, and a great number of the included meta-analyses reported no funding $(n=80,37.4 \%)$. The median number of authors was 5 (IQR 4-6). 
Table 2. Epidemiological characteristics of meta-analyses included in the study

\begin{tabular}{|c|c|c|}
\hline Journal & $\mathrm{n}$ & $\%$ \\
\hline General & 55 & $25.7 \%$ \\
\hline Specialty & 159 & $74.3 \%$ \\
\hline \multicolumn{3}{|l|}{ Country } \\
\hline Brazil & 61 & $28.5 \%$ \\
\hline USA & 24 & $11.2 \%$ \\
\hline China & 18 & $8.4 \%$ \\
\hline United Kingdon & 11 & $5.1 \%$ \\
\hline Switzerland & 9 & $4.2 \%$ \\
\hline Germany & 8 & $3.7 \%$ \\
\hline Sweden & 7 & $3.2 \%$ \\
\hline Other 30 countries and unclear & 76 & $35.7 \%$ \\
\hline \multicolumn{3}{|l|}{ Dental Specialties } \\
\hline Oral and Maxillofacial Surgery & 32 & $14.9 \%$ \\
\hline Implantology & 31 & $14.5 \%$ \\
\hline Periodontics & 29 & $13.5 \%$ \\
\hline Orthodontics & 26 & $12.1 \%$ \\
\hline Oral and Maxillofacial Pathology & 24 & $11.2 \%$ \\
\hline Restorative and Esthetic Dentistry & 19 & $8.8 \%$ \\
\hline Pediatric Dentistry & 17 & $7.9 \%$ \\
\hline Endodontics & 13 & $6.1 \%$ \\
\hline Public Health & 12 & $5.6 \%$ \\
\hline Prosthodontics & 5 & $2.3 \%$ \\
\hline Radiology & 5 & $2.3 \%$ \\
\hline Others & 1 & $0.5 \%$ \\
\hline \multicolumn{3}{|l|}{ Focus } \\
\hline Treatment/Therapeutic & 101 & $47.20 \%$ \\
\hline Diagnosis & 41 & $19.2 \%$ \\
\hline Epidemiology & 29 & $13.5 \%$ \\
\hline Prognosis & 22 & $10.3 \%$ \\
\hline Prevention & 9 & $4.2 \%$ \\
\hline Other & 6 & $2.8 \%$ \\
\hline Unclear & 6 & $2.8 \%$ \\
\hline \multicolumn{3}{|l|}{ Funding } \\
\hline No funding & 80 & $37.4 \%$ \\
\hline Not reported & 77 & $36 \%$ \\
\hline Non-profit sponsor & 53 & $24.8 \%$ \\
\hline Unclear & 3 & $1.4 \%$ \\
\hline Mixed & 1 & $0.5 \%$ \\
\hline Number of authors & \multicolumn{2}{|c|}{ Median $5(\mathrm{IQR}=4-6)$} \\
\hline
\end{tabular}

IQR - Interquartile range 
Table 3 presents the conduct and reporting characteristics of meta-analyses. Most of the SRs reported that a meta-analysis was conducted in the title $(n=187,87.4 \%)$. The "continuous variable" was the type of outcome most used ( $n=41,34.4 \%)$, and the unit of measure of the first reported result of the primary outcome was "mean difference" ( $n=45,38.5 \%)$. Most of the meta-analyses reported the statistical significance of the first reported result of the primary outcome as "favorable, statistically significant" ( $n=60,50.8 \%$ ), and the majority of studies used a random effect model for all meta-analyses $(n=130,62.5 \%)$. Regarding the statistical heterogeneity of included studies in the meta-analysis, the majority of meta-analyses described some method to formally evaluate the statistical heterogeneity of included studies ( $n=180,87.0 \%)$, and the method most used was the $\mathrm{I}^{2}$ test $(\mathrm{n}=161,75.2 \%)$. When additional analyses were conducted, the most used was subgroup ( $n=65,30.4 \%)$; however, most of the meta-analyses did not conduct additional analyses $(n=121,56.6 \%)$. The software most used to conduct meta-analyses was RevMan ( $n=73,34.1 \%)$, followed by Stata $(n=34$, $15.9 \%$ ). The median of studies included in the largest meta-analyses was 9 (IQR 5-16). Forty (40) meta-analyses (18.7\%) presented a funnel plot graph; however, 10 did not report that the analysis was performed, and a further 3 did not report that publication bias was assessed.

Table 3. Characteristics of conduct and reporting of meta-analyses

\begin{tabular}{|c|c|c|}
\hline Terms in the title & $\mathrm{n}$ & $\%$ \\
\hline Systematic review and meta-analysis & 160 & $74.8 \%$ \\
\hline Only meta-analysis & 27 & $12.6 \%$ \\
\hline Only systematic review & 17 & $7.9 \%$ \\
\hline Neither & 10 & $4.7 \%$ \\
\hline \multicolumn{3}{|c|}{ Did the review authors specify one or more primary outcome(s)? } \\
\hline Yes & 106 & $50.7 \%$ \\
\hline No & 90 & $43.1 \%$ \\
\hline No but only one outcome reported & 13 & $6.2 \%$ \\
\hline \multicolumn{3}{|c|}{ What type of outcome is the primary outcome? } \\
\hline Continuous & 41 & $34.4 \%$ \\
\hline Dichotomous & 25 & $21 \%$ \\
\hline Both dichotomous and continuous & 23 & $19.3 \%$ \\
\hline Not reported & 15 & $12.6 \%$ \\
\hline Rate & 12 & $10.1 \%$ \\
\hline Unclear & 3 & $2.5 \%$ \\
\hline \multicolumn{3}{|c|}{ What is the unit of measure of the first reported result (effect estimate) of the primary outcome? } \\
\hline Mean difference & 45 & $38.5 \%$ \\
\hline Risk ratio & 24 & $20.5 \%$ \\
\hline Odds ratio & 18 & $15.4 \%$ \\
\hline Standardized mean difference & 12 & $10.3 \%$ \\
\hline Prevalence & 6 & $5.1 \%$ \\
\hline
\end{tabular}


Continuation

\begin{tabular}{lll}
\hline Not reported or unclear & 6 & $5.1 \%$ \\
\hline Likelihood ratios & 5 & $4.3 \%$ \\
\hline Event Rate & 1 & $0.8 \%$
\end{tabular}

What is the statistical significance of the first reported result (effect estimate) of the primary outcome?

\begin{tabular}{lll}
\hline Favorable, statistically significant & 60 & $50.8 \%$ \\
\hline Favorable, non-statistically significant & 19 & $16.1 \%$ \\
\hline Unfavorable, non-statistically significant & 14 & $11.9 \%$ \\
\hline Unfavorable, statistically significant & 12 & $10.2 \%$ \\
\hline Non comparative & 12 & $10.2 \%$ \\
\hline Not reported & 1 & $0.8 \%$ \\
\hline Which meta-analysis model was used in the meta-analyses? & & \\
\hline Random-effects model for all meta-analyses & 130 & $62.5 \%$ \\
\hline Varied & 47 & $22.6 \%$ \\
\hline Fixed-effect model for all meta-analyses & 21 & $10.1 \%$ \\
\hline Other & 3 & $1.4 \%$ \\
\hline Not reported & 7 & $3.4 \%$ \\
\hline
\end{tabular}

Was the risk of bias (or quality) assessment incorporated into any meta-analyses in the review?

\begin{tabular}{lcc}
\hline No & 119 & $59.9 \%$ \\
\hline Yes & 79 & $40.1 \%$
\end{tabular}

Was any method described to formally evaluate statistical heterogeneity of included studies?

\begin{tabular}{|c|c|c|}
\hline No & 25 & $12.1 \%$ \\
\hline Yes & 180 & $87 \%$ \\
\hline $\begin{array}{l}\text { Statistical heterogeneity was not taken into account } \\
\text { using formal statistical evaluation, but heterogeneity } \\
\text { of the studies was qualitatively assessed }\end{array}$ & 2 & $0.9 \%$ \\
\hline \multicolumn{3}{|c|}{$\begin{array}{l}\text { Which methods were used to formally evaluate statistical heterogeneity of included studies? (*considering } \\
214 \text { studies) }\end{array}$} \\
\hline Chi-square or Cochran's Q & 109 & $50.9 \% *$ \\
\hline I^2 (I-square) & 161 & $75.2 \% *$ \\
\hline tau^2 (tau-square) & 17 & $7.9 \%$ \\
\hline Other & 6 & $2.8 \% *$ \\
\hline
\end{tabular}

Did the authors report that a measure of statistical heterogeneity was used to justify use of a fixed-effect or random-effects meta-analysis model?

\begin{tabular}{lcc}
\hline No & 81 & $41.5 \%$ \\
\hline Yes & 114 & $58.5 \%$ \\
\hline
\end{tabular}

Did the authors report assessing (or an intent to assess) publication bias?

\begin{tabular}{|c|c|c|}
\hline $\begin{array}{l}\text { No, publication bias was not assessed, and the } \\
\text { authors did not report an intention to assess it }\end{array}$ & 106 & $49.5 \%$ \\
\hline $\begin{array}{l}\text { No, publication bias was not assessed, but the } \\
\text { authors reported that they intended to assess it if they } \\
\text { identified a sufficient number of studies }\end{array}$ & 29 & $13.5 \%$ \\
\hline Yes, publication bias was assessed & 79 & $36.9 \%$ \\
\hline
\end{tabular}


Continuation

\begin{tabular}{|c|c|c|}
\hline \multicolumn{3}{|c|}{$\begin{array}{l}\text { Which methods did the authors report using (or intending to use) to assess publication bias? (*considering } \\
\text { the number total of studies) }\end{array}$} \\
\hline Begg's test & 6 & $2.8 \% *$ \\
\hline Egger's test & 25 & $11.7 \% *$ \\
\hline Funnel plot & 64 & $30 \% *$ \\
\hline $\begin{array}{l}\text { Sensitivity analysis comparing fixed-effect to random- } \\
\text { effects model }\end{array}$ & 3 & $1.4 \% *$ \\
\hline Subgroup analyses by sample size & 2 & $0.9 \% *$ \\
\hline Other & 2 & $0.9 \% *$ \\
\hline $\begin{array}{l}\text { Funnel plot presented }\left({ }^{*} \text { considering the number total }\right. \\
\text { of studies) }\end{array}$ & 40 & $18.7 \%$ \\
\hline \multicolumn{3}{|c|}{ Which of the following additional analyses did the authors conduct? } \\
\hline Meta-regression & 21 & $9.8 \%$ \\
\hline Network meta-analysis & 2 & $0.9 \%$ \\
\hline Sensitive & 41 & $19.2 \%$ \\
\hline Subgroup & 65 & $30.4 \%$ \\
\hline No additional analyses & 121 & $56.6 \%$ \\
\hline \multicolumn{3}{|c|}{ What statistical software was used to perform meta-analyses? } \\
\hline RevMan & 73 & $34.1 \%$ \\
\hline Stata & 34 & $15.9 \%$ \\
\hline Comprehensive Meta-Analysis & 31 & $14.5 \%$ \\
\hline $\mathrm{R}$ & 18 & $8.4 \%$ \\
\hline Not reported & 18 & $8.4 \%$ \\
\hline Others & 13 & $6.1 \%$ \\
\hline Two or more different programs & 13 & $6.1 \%$ \\
\hline MedCalc & 8 & $3.7 \%$ \\
\hline OpenMeta & 6 & $2.8 \%$ \\
\hline Median of studies included in the largest meta-analysis & & \\
\hline
\end{tabular}

For the primary outcome, 90 meta-analyses (43.1\%) did not specify the primary outcome, and most of the meta-analyses did not incorporate the risk of bias assessment into them $(n=119,59.9 \%)$. Most of the meta-analyses reported that a measure of statistical heterogeneity was used to justify the use of a fixed-effect or random-effects meta-analysis model $(n=114,58.5 \%)$ and did not assess publication bias $(n=106,49.5 \%)$.

\section{Discussion}

Our study is the first in dentistry to assess how meta-analyses are conducted and reported. We identified that the reporting and conduct characteristics of meta-analyses are varied. Most of the studies reported that a meta-analysis was conducted in the title and formally evaluated the statistical heterogeneity of included studies. However, most of the meta-analyses reported that a measure of statistical heterogeneity was 
used to justify the use of a fixed-effect or random-effects meta-analysis model and did not assess publication bias, thus demonstrating that there is room for improvement in conducting meta-analyses in dentistry. In addition, our estimates showed an increase rate of SRs with meta-analyses published in recent years compared to a previous study 5 .

Several studies in dentistry assessed the quality of meta-analysis published in different dental specializations ${ }^{11,12}$. Both publications demonstrated that the meta-analysis quality can vary from medium to low and highlighted that important aspects such as publication bias assessment were not conducted in most meta-analyses; these findings were also corroborated by our study. Page et al. ${ }^{4}$ demonstrated that flaws in meta-analyses are not restricted to dentistry. The authors evaluated 110 statistical analyses in systematic reviews of therapeutic interventions in the biomedical field and pointed out that the involvement of statisticians in conducting systematic reviews could improve the quality of meta-analyses.

Three important flaws in the conduct/report of meta-analyses were identified. First, almost $50 \%$ of the included studies did not assess the impact of publication bias. Publication bias occurs when there is a predilection for publishing particular results, such as in studies reporting high effect sizes which are more likely to be published than studies reporting lower effect sizes, and this type of bias can affect systematic reviews. Failure to assess the possible impact of publication bias on systematic reviews results can generate an invalid estimate, which could guide interventions or actions based on biased conclusions ${ }^{1,13}$. Koletsi et al. ${ }^{14}$ (2016) assessed 162 systematic reviews in dentistry, with the findings demonstrating a low rate of publication bias analysis performed and a high rate of improperly conducted publication bias assessment.

The second important flaw is the use of a statistical heterogeneity measure to justify the use of a fixed- or random-effects meta-analysis model. This could be considered a shortcoming since the choice of each effect only based on a statistical measure could exacerbate the effect of bias, thus producing a spurious estimate and an inappropriate conclusion. Recent recommendations clearly affirm that the decision between the use of the model is a topic of much debate. An alternative to the topic is a pragmatic approach planned to perform both analyses (fixed and random models), while the random effect model can be present in cases of not identifying asymmetry in funnel plot, even knowing that funnel plot asymmetry suggests that both methods are problematic. In addition, the author pointed out that "the choice between a fixed-effect and a random-effects meta-analysis should never be made based on a statistical test for heterogeneity"1.

Lastly, almost 50\% of the meta-analyses included did not specify the primary outcome. Tricoo et al. ${ }^{15}$ (2016) evaluated a sample of 96 systematic reviews and demonstrated that one-third of the studies did not specify or change the primary outcome. We did not assess if the studies included in our study were registered or did not compare the information reported in protocols and in the final publication, however it could be possible that study results were related to outcome reporting bias, generating imprecise results. 
There are some undeniable limitations of our study. We only considered one database and only studies published in English, and we performed a cross-sectional analysis only considering 2017. Also, our data extraction was based on the study report, and it is possible that some SRs were conducted more rigorously than was specified in the report. Additionally, we did not include meta-analyses without a systematic review, which could have led to selecting studies with high methodological quality, and we did not judge the study quality and if a correct methodology was used to perform the meta-analyses because this was not the aim of this study.

Various approaches are encouraged to minimize the flaws identified in our analysis: 1) graduate program students should be receiving adequate training to perform and report meta-analyses; 2) involvement of statisticians on the systematic review team could improve analysis quality; 3) dissemination of PRISMA Statement extensions such as PRISMA for Individual Patient Data and Network Meta-Analyses could help in reporting adequate meta-analyses.

In conclusion, we identified deficiencies in the reporting and conduct of meta-analyses in dentistry, suggesting that there is room for improvement. Educational approaches are necessary to improve the quality of such analyses and to avoid unbiased and imprecise results.

\section{Acknowledgements}

BAA, and RSO are funded in part by Meridional Foundation (Passo Fundo - Brazil). LL and LD are supported by CAPES, Brazil. This study was conducted in a Graduate Program supported by CAPES, Brazil (Finance Code 001). The funders had no role in the study design, data collection and analysis, decision to publish or preparation of the manuscript.

\section{References}

1. Page MJ, Higgins JPT, Sterne JAC. Assessing risk of bias due to missing results in a synthesis. In: Higgins J, Thomas J. Cochrane handbook for systematic reviews of interventions. Cochrane; 2019. Version 6.0. Chapter 13.

2. McKenzie JE, Beller EM, Forbes AB. Introduction to systematic reviews and meta-analysis. Respirology. 2016 May;21(4):626-37. doi: 10.1111/resp.12783.

3. Ioannidis JP. The mass production of redundant, misleading, and conflicted systematic reviews and meta-analyses. Milbank Q. 2016 Sep;94(3):485-514. doi: 10.1111/1468-0009.12210.

4. Page MJ, Altman DG, McKenzie JE, Shamseer L, Ahmadzai N, Wolfe D, et al. Flaws in the application and interpretation of statistical analyses in systematic reviews of therapeutic interventions were common: a cross-sectional analysis. J Clin Epidemiol. 2018 Mar;95:7-18. doi: 10.1016/j.jclinepi.2017.11.022

5. Saltaji H, Cummings GG, Armijo-Olivo S, Major MP, Amin M, Major PW, et al. A descriptive analysis of oral health systematic reviews published 1991-2012: cross sectional study. PLoS One. 2013 Sep;8(9):e74545. doi: 10.1371/journal.pone.0074545.

6. Jayaraman J, Nagendrababu V, Pulikkotil SJ, Innes NP. Critical appraisal of methodological quality of Systematic Reviews and Meta-analysis in Paediatric Dentistry journals. Int J Paediatr Dent. 2018 Nov;28(6):548-560. doi: 10.1111/ipd.12414. 
7. Bassani R, Pereira GKR, Page MJ, Tricco AC, Moher D, Sarkis-Onofre R. Systematic reviews in dentistry: Current status, epidemiological and reporting characteristics. J Dent. 2019 Mar;82:71-84. doi: 10.1016/j.jdent.2019.01.014.

8. Moher D, Shamseer L, Clarke M, Ghersi D, Liberati A, Petticrew M, et al. Preferred reporting items for systematic review and meta-analysis protocols (PRISMA-P) 2015 statement. Syst Rev. 2015 Jan;4(1):1. doi: 10.1186/2046-4053-4-1.

9. Liberati A, Altman DG, Tetzlaff J, Mulrow C, Gøtzsche PC, Ioannidis JP, et al. The PRISMA statement for reporting systematic reviews and meta-analyses of studies that evaluate healthcare interventions: explanation and elaboration. BMJ. 2009 Jul;339:b2700. doi: 10.1136/bmj.b2700.

10. Page MJ, Shamseer L, Altman DG, Tetzlaff J, Sampson M, Tricco AC, et al. Epidemiology and reporting characteristics of systematic reviews of biomedical research: a cross-sectional study. PLoS Med. 2016 May;13(5):e1002028. doi: 10.1371/journal.pmed.1002028.

11. Kattan S, Lee SM, Kohli MR, Setzer FC, Karabucak B. methodological quality assessment of meta-analyses in endodontics. J Endod. 2018 Jan;44(1):22-31. doi: 10.1016/j.joen.2017.07.019.

12. El-Rabbany M, Li S, Bui S, Muir JM, Bhandari M, Azarpazhooh A. A Quality analysis of systematic reviews in dentistry, part 1: meta-analyses of randomized controlled trials. J Evid Based Dent Pract. 2017 Dec;17(4):389-98. doi: 10.1016/j.jebdp.2017.06.004.

13. Thornton A, Lee P. Publication bias in meta-analysis: its causes and consequences. J Clin Epidemiol. 2000 Feb;53(2):207-16. doi: 10.1016/s0895-4356(99)00161-4.

14. Koletsi D, Valla K, Fleming PS, Chaimani A, Pandis N. Assessment of publication bias required improvement in oral health systematic reviews. J Clin Epidemiol. 2016 Aug;76:118-24. doi: 10.1016/j.jclinepi.2016.02.019.

15. Tricco AC, Cogo E, Page MJ, Polisena J, Booth A, Dwan K, et al. A third of systematic reviews changed or did not specify the primary outcome: a PROSPERO register study. J Clin Epidemiol. 2016 Nov;79:46-54. doi: 10.1016/j.jclinepi.2016.03.025. 